\title{
Learning and generation of temporal sequences in the neocortex
}

\author{
Sergio Verduzco-Flores ${ }^{1 *}$, Mark Bodner ${ }^{1,2}$, Bard Ermentrout $^{1}$ \\ From Nineteenth Annual Computational Neuroscience Meeting: CNS*2010 \\ San Antonio, TX, USA. 24-30 July 2010
}

The temporal structure of neuronal activity plays a fundamental role in brain function. In addition to the compelling structure found in birdsong, repeating temporal sequences have been experimentally observed in the mammalian neocortex, both at the levels of local field potentials and individual neurons.

The mechanisms underlying the learning and generation of temporal sequences are currently unknown. An attractive idea is that time-asymmetric Hebbian mechanisms capture the temporal structure of afferent signals by selectively strengthening the connections between sequentially activated neuronal populations. We explore some consequences of this idea using a simplified model of neocortex.

Our model uses excitatory and inhibitory firing rate variables, along with adaptation and time-asymmetric Hebbian plasticity to create a versatile pattern generator which can store and reconstruct input sequences. We study several related properties of this model, mainly: 1 ) the formation of intersecting and complex sequences, 2) how the structure in the connection matrix affects the dynamics of the system and the symmetries observed in the activity of the network, 3) pathological behaviors due to abnormalities in plasticity and inhibition; the possible relation with epilepsy.

\section{Author details}

'Department of Mathematics, University of Pittsburgh, Pittsburgh, Pennsylvania 15260, USA . ${ }^{2}$ MIND Research Institute, Santa Ana, California 92704, USA.

- No space constraints or color figure charges

- Immediate publication on acceptance

- Inclusion in PubMed, CAS, Scopus and Google Scholar

- Research which is freely available for redistribution

"Correspondence: sov2@pitt.edu
'Department of Mathematics, University of Pittsburgh, Pittsburgh, Pennsylvania 15260, USA 